\title{
Value of Fragmented QRS Complex on 12-lead ECG as a Valuable Marker of Myocardial Damage of CAD Patients
}

\author{
Andrico L. Tobing, Anggia Chairuddin Lubis, Edison Bun, Harris Hasan
}

\begin{abstract}
Background: Presence of Fragmented QRS (fQRS) on a I2-lead Electrocardiogram (ECG) was associated with various cardiac diseases. This phenomenon could represent as an electrical disruption of conduction system following myocardial damage due to coronary artery disease (CAD). We aimed to investigate the value of fQRS to detect the myocardial scar as detected by SPECT Myocardial Perfusion Imaging (MPI).

Methods: A cross-sectional study of patients who were either being evaluated for CAD or had a history of CAD and underwent Cardiac SPECT. The fQRS defined as morphologies of QRS wave $(<I 20 \mathrm{~ms})$, which included an additional $R$ wave ( $\left.R^{\prime}\right)$, notching in the nadir of $S$ wave, or $>$ I R' (fragmentation) in 2 contiguous leads, corresponding to a major coronary artery territory. Pathological $\mathrm{Q}$ wave, paced rhythm, typical right or left bundle branch block pattern with QRS duration of $\geq 120 \mathrm{~ms}$ were excluded. MPI was interpreted by visual analysis and semi-quantitative scores on 17-segment assessment according to standard nomenclature.

Results: Total of 72 patients ( 49 males, mean age $54.7 \pm 9.8$ years). fQRS was found in 46 patients (64\%). The frequency of myocardial scar was significantly higher in patients with fQRS ( $89 \%$ vs. I5\%, p<0.05). Sensitivity, specificity, positive and negative predictive value of fQRS for any of myocardial scar as detected by SPECT analysis were $91 \%, 81 \%, 89 \%$, and $84 \%$, respectively. From regional scar analysis, fQRS has sensitivity and specificity of $87 \%$ and $90 \%$ for anterior wall, $76 \%$ and $80 \%$ for inferior wall, $73 \%$ and $79 \%$ for lateral wall. LVEF was significantly lower in patients with fQRS (36.9 \pm 2 .I vs. $53.2 \pm 2.2, p<0.05)$.

Conclusion: The fragmented QRS could serve as an important ECG marker to detect and localize the myocardial damage in patients with known or suspected CAD. Regional fQRS patterns denote the presence of regional myocardial scar and are a valuable diagnostic marker of CAD with good sensitivity and specificity.
\end{abstract}

Keywords: fragmented QRS, SPECT

(Indonesian J Cardiol. 2019;40:249-257)

Departemen Kardiologi dan Kedokteran Vaskular FK USU/RSUP

\section{Introduction}

Haji Adam Malik, Medan, Indonesia

Correspondence:

dr. Andrico N. L. Tobing.

Departemen Kardiologi dan Kedokteran Vaskular FK USU/RSUP

Haji Adam Malik, Medan, Indonesia

E-mail: holladoctor@gmail.com

oronary artery disease (CAD) is known as
a leading cause of death in many countries.
In United States, it is estimated more than
17 million people suffered from CAD ${ }^{1}$.

Meanwhile in Indonesia, according to RISKESDAS 2013, prevalence of CAD reaches $1,5 \%$ of the entire 
population, and is expected to continue to rise in coming years $^{2}$. The considerably high global incidence of CAD with its impact on morbidity, mortality, and treatment costs shows the importance of simple, accessible, and cost-effective diagnostic tools. The most universal modality that meets almost all of the mentioned attributes is electrocardiography (ECG) ${ }^{3}$.

Electrocardiogram (ECG) is the first useful diagnostic tool, readily available, and commonly used to diagnose coronary artery disease. Previous studies have shown that any change in QRS wave morphology is related to ventricular conduction disturbance and could be caused by myocardial damage due to ischemia or infarction of the ventricle ${ }^{4}$. Previously, pathological Q wave on a 12lead ECG is a well-known marker for detecting prior Myocardial Infarction (MI) but some studies found that Q-waves do not always appear on post-MI ECG and can regress or even disappear over time in $25-60 \%$ of patients 5 . Thus, an increasing interest has developed in fragmented QRS (fQRS) and its relationship with previous MI. Some researchers have claimed that fQRS might show regional myocardial damage in non-Q wave MI patients and is associated with a higher all-cause mortality and cardiac event rate ${ }^{6,7}$.

Fragmentation of QRS may represent a heterogeneous left ventricular activation state. The presence of left ventricular depolarization changes may manifest as fQRS appearance on 12-lead ECG ${ }^{8}$. The existence of a preliminary deflection of visible $\mathrm{R}$ waves can occur as result of a shift from the QRS wave vector to the right and posterior because of early depolarization process. The presence of a notch or a slurred of the $S$ wave may represent the activation pattern of the precordial lead (to the left of the transition zone), similar to the activation pattern of the left bundle branch block. Therefore, the delayed conduction of QRS waves arising from the presence of block or slowing of the conduction pathway will lead to fragmented QRS pattern?.

The fragmentation of the QRS complex on 12 leads ECG examination could be found in many heart diseases such as ventricular aneurysm, idiopathic dilated cardiomyopathy, myocardial fibrosis, sarcoidosis, Brugada syndrome, arrhythmogenic right ventricular cardiomyopathy and myocarditis ${ }^{10}$. The incidence of fQRS in CAD patients is significantly associated with left ventricular dysfunction and decreased perfusion of the myocardium. The presence of QRS fragmentation in the ECG is associated with the existence of ischemia, infarct or fibrosis and results from damage to the signal transduction and depolarization processes in the Purkinje fiber of the ventricles 7,11 . Fragmentation of QRS may originate from an abnormal area of myocardium where ventricular activation is delayed and asynchronous which causes notching in the QRS complex ${ }^{12}$. Nevertheless, this warrants further investigation because fragmented QRS) is a relatively new marker with contradictory results reported in different studies.

Single-Photon Emission Computed Tomography (SPECT) is an accurate non-invasive modality for investigation of coronary artery disease with high sensitivity and specificity, and it also has a good prognostic value ${ }^{13}$. The quantitative analysis of myocardial wall movement abnormalities using SPECT is an accurate modality for assessing the presence of severe and extensive coronary artery disease ${ }^{14}$.

Stress Myocardial Perfusion Imaging (MPI) by Technetium-99m sestamibi-based Single Photon Emission Computed Tomography is routinely performed to identify the extent of a prior myocardial injury and the presence of physiologically significant myocardial ischemia in patients with known or suspected coronary artery disease ${ }^{15}$. Stress SPECT-based technique has also demonstrated superior accuracy and clinical utility in identifying the presence and size of myocardial infarction scar ${ }^{11,15}$.

We aimed to investigate the value of Fragmented QRS complex to detect and localize the myocardial scar in patients with coronary artery disease by using Stress Myocardial Perfusion Imaging (MPI) with Technetium$99 \mathrm{~m}$ sestamibi-based Single Photon Emission Computed Tomography (SPECT).

\section{Methods}

\section{Study design and population}

This is a cross-sectional study that included patients who were either being evaluated for CAD or had a history of CAD who underwent SPECT Myocardial Perfusion Imaging at Adam Malik General Hospital from Januari 2017 to March 2018. We conducted data collection through anamnesis, physical examination, laboratory examination, ECG, cardiac exercise test, echocardiography, and other supporting modalities. The 


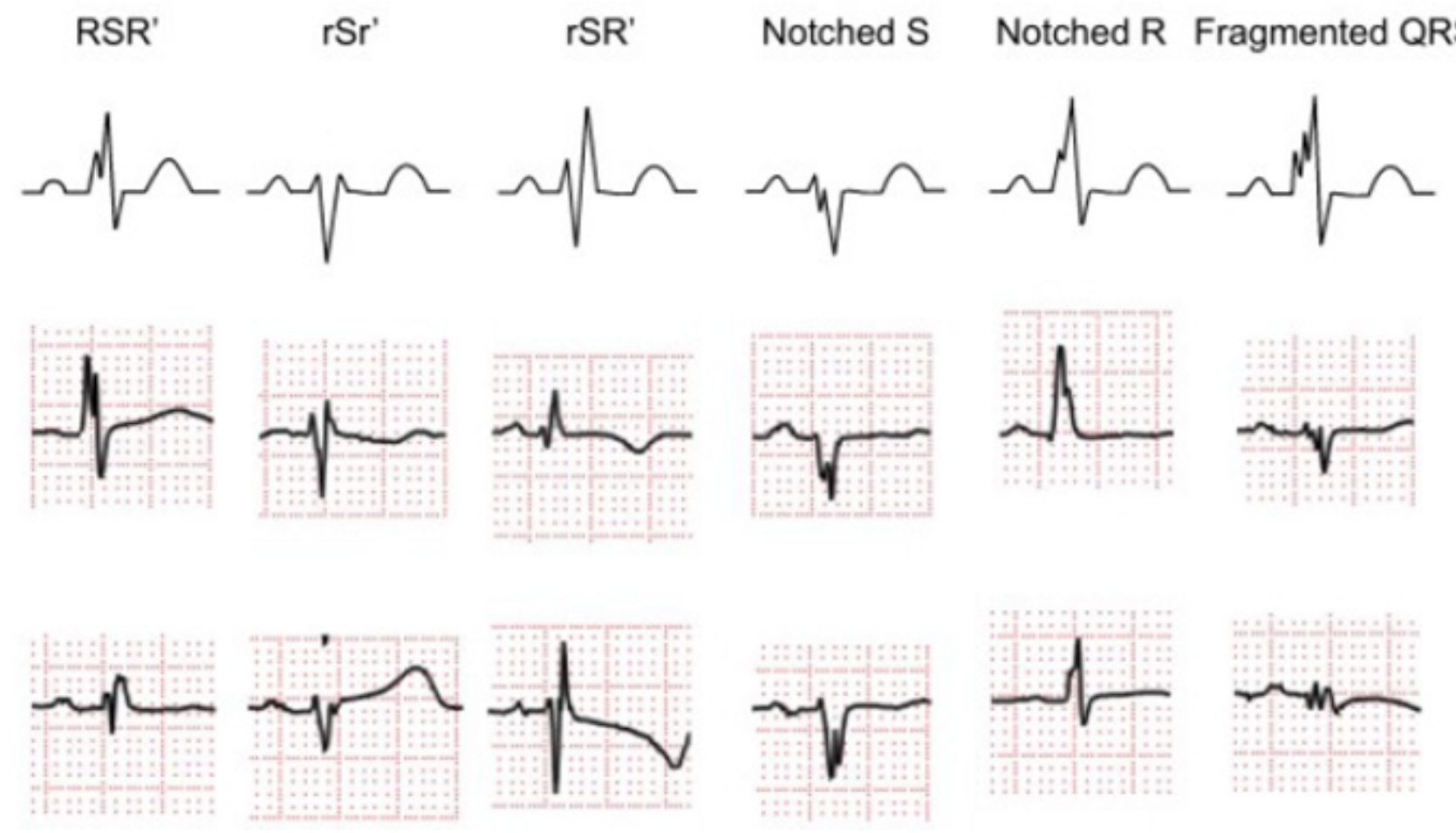

Figure 1. Various morphologies of QRS fragmentation on a 12-lead ECG ${ }^{11}$

demographics of all patients, including risk factors for CAD (age, sex, hypertension, smoking, type II diabetes mellitus, dyslipidemia) were recorded.

Exclusion criteria were ECG findings of pathological Q wave, paced rhythm, typical right or left bundle branch block pattern with QRS duration of $\geq 120 \mathrm{~ms}$ and patients who are unable to complete an optimal SPECT examination. Written informed consent was obtained from all patients before the SPECT study. The study protocol was approved by the ethics committee of the University.

\section{ECG criteria for Fragmented QRS}

The measurement of QRS complex fragmentation was performed at just before the SPECT examination was conducted. Resting baseline 12-lead ECG $(100 \mathrm{~Hz}$, $25 \mathrm{~mm} / \mathrm{s}, 10 \mathrm{~mm} / \mathrm{mV}$; Fukuda Denshi, Tokyo, Japan, model FX-8322 R) was obtained and analyzed by two independent readers who were blinded to the SPECT findings. The fragmented QRS defined as morphologies of QRS wave $(<120 \mathrm{~ms})$, which included an additional $R$ wave ( $R$ '), notching in the nadir of $S$ wave, or $>1$ ' (fragmentation) in 2 contiguous leads, corresponding to a major coronary artery territory (Figure 1$)^{11}$. The presence of $\mathrm{fQRS}$ in $\geq 2$ contiguous anterior leads (V1 to V5) was assigned to myocardial scar in anterior segments or in the left anterior descending territory. The lateral leads (I, aVL, and V6) was assigned to myocardial scar in lateral segments or left circumflex territory. The inferior leads (II, III, and aVF) was assigned to myocardial scar in the inferior segments or in the right coronary artery territory.

\section{SPECT Acquisition and Analysis}

Patients were advised not to take beta-blockers, calcium channel blockers, aminophylline or caffeine 24-hour before the test. All patients underwent a rest/stress (lowdose/high-dose) Technetium-99m sestamibi single-day stress protocol. Dobutamine was used as pharmacologic stress agents. Dobutamine was infused at incremental doses of 5,10,20, 30 and $40 \mathrm{mg} / \mathrm{kg}$ per minute at 3 -min intervals, until symptoms or target heart rate is achieved. Patients who are unable to complete an optimal cardiac SPECT examination (severe complaints, hemodynamic deterioration or malignant ECG changes during examination) were also be excluded. 
Table 1. Baseline characteristics

\begin{tabular}{lccc}
\hline \multirow{2}{*}{ Variables } & \multicolumn{2}{c}{$\begin{array}{c}\text { Fragmented QRS } \\
\text { (fQRS) }\end{array}$} & \\
\cline { 2 - 3 } & $\begin{array}{c}\text { With } \\
(\mathrm{n}=46)\end{array}$ & $\begin{array}{c}\text { Without } \\
(\mathrm{n}=26)\end{array}$ & \\
\hline Age (mean \pm SD), years & $56,2 \pm 8,4$ & $51,9 \pm 11,5$ & $0,1^{\mathrm{a}}$ \\
Gender (n, \%) & & & \\
Male & $38(82)$ & $11(42)$ & $0,001^{\mathrm{b}}$ \\
Female & $8(18)$ & $15(58)$ & \\
CAD risk factors (n, \%) & & & \\
Hypertension & $22(48)$ & $12(46)$ & $0,8^{\mathrm{b}}$ \\
Type 2 Diabetes & $26(56)$ & $8(30)$ & $0,03^{\mathrm{b}}$ \\
Mellitus & $39(84)$ & $17(65)$ & $0,06^{\mathrm{b}}$ \\
Dyslipidemia & $38(82)$ & $13(50)$ & $0,003^{\mathrm{b}}$ \\
Smoker & & & $0,8^{\mathrm{b}}$ \\
Post PCI & $6(13)$ & $3(11)$ & $0,29^{\mathrm{c}}$ \\
Post CABG & $4(8)$ & $0(0)$ & 0
\end{tabular}

$\mathrm{SD}=$ Standard Deviation; $\mathrm{PCI}=$ Percutaneous Coronary Intervention; $\mathrm{CAD}=$ Coronary Artery Disease; $\mathrm{CABG}=$ Coronary Artery Bypass Grafting; aStudent's t-test; bChi Square Test; cFisher's Exact Test

Table 2. SPECT analysis results between groups

\begin{tabular}{lccc}
\hline \multirow{2}{*}{ Variables } & \multicolumn{2}{c}{ Fragmented QRS } & \multirow{2}{*}{ P value } \\
\cline { 2 - 3 } & With & Without & \\
\hline LVEF (\%) & $36,9 \pm 2,1$ & $53,2 \pm 2,2$ & 0,002 \\
Rest LVESV (mean \pm SD), ml & $138,1 \pm 82$ & $97,5 \pm 64,1$ & $0,02 \mathrm{a}$ \\
Stress LVESV (mean $\pm S D), \mathrm{ml}$ & $139 \pm 86,1$ & $91 \pm 57,6$ & $0,006^{\mathrm{a}}$ \\
Myocardial scar, $\mathrm{n}(\%)$ & $41(89)$ & $4(15)$ & $<0,001$ \\
\hline
\end{tabular}

$\overline{\mathrm{SD}}=$ Standard Deviation; LVEF $=$ Left Ventricle Ejection Fraction; LVESV = Left Ventricle End Systolic Volume; aStudent's t-test

The ECG-gated acquisition was performed on poststress images at 8 frames per R-R interval, acquired within 20 to 45 minutes after peak stress Tc-99m administration. Gated SPECT images were obtained with ECG-gated rotating, $90^{\circ}$ fixed dual-head gamma camera (Philips Medical System, Cleveland). Tomograms were reconstructed by different axis, including projection in vertical long-axis, horizontal long-axis, and short-axis planes. Motion correction software was used when needed, as determined by the reader. The rest and stress acquisitions were analyzed using an automated AutoQuant software (Cedars-Sinai Medical Center, Los Angeles, California) (Figure 2).

SPECT images were evaluated visually and semiquantitatively by nuclear medicine specialists who was an independent blinded reader. A semiquantitative Sum Stress Score (SSS), Sum Rest Score (SRS), and Sum Difference Score (SDS) were calculated on 17-segment analysis using 5 -point scale $(0=$ normal, $1=$ equivocal or mildly abnormal, 2 = moderately abnormal, $3=$ severely abnormal, and $4=$ absent tracer uptake). Individual epicardial coronary artery regional segments were scored according to standard nomenclature ${ }^{16}$. The left anterior descending artery (7 segments) represented by anterior leads; the left circumflex artery ( 5 segments) represented by lateral leads; and the right coronary artery ( 5 segments) represented by inferior lead (Figure 3). A myocardial scar was defined by a total regional SSS and $S R S \geq 3$ and a regional $S D S \leq 2$, corresponding to a major coronary artery region.

\section{Statistical Analysis}

Categorical variables were presented as the number or frequency $(\mathrm{n})$ and percentage (\%). Continuous variables were expressed as mean and standard deviation (SD). Categorical variables were analyzed by using Chisquared $\left(\chi^{2}\right)$ and Fisher's exact test whereas continuous variables were analyzed by Independent $T$ or MannWhitney test. Normality test was done using one sample Kolmogorov-Smirnov $(\mathrm{n}>50)$ or the Shapiro-Wilk ( $<50$ ). Sensitivity was defined as the number of truepositive tests divided by the total number of patients with myocardial scar defined by SPECT. Specificity was defined as the number of true-negative tests divided by the total number of patients without myocardial scar. Positive and negative predictive values were also calculated. Positive predictive value is the probability that subjects with a positive screening test truly have the disease and calculated as (true positive)/(true positive + false positive). Negative predictive value is the probability that subjects with a negative screening test truly do not have the disease and calculated as (true negative) $/($ false negative + true negative). P-value $<0,05$ is considered to be statistically significant. All statistical analysis were done using SPSS software for Windows.

\section{Results}

86 patients were enrolled in our study, 14 patients (16\%) were excluded from our analysis because of pathological Q wave $(n=6)$, typical RBBB or LBBB $(n=3)$, and 


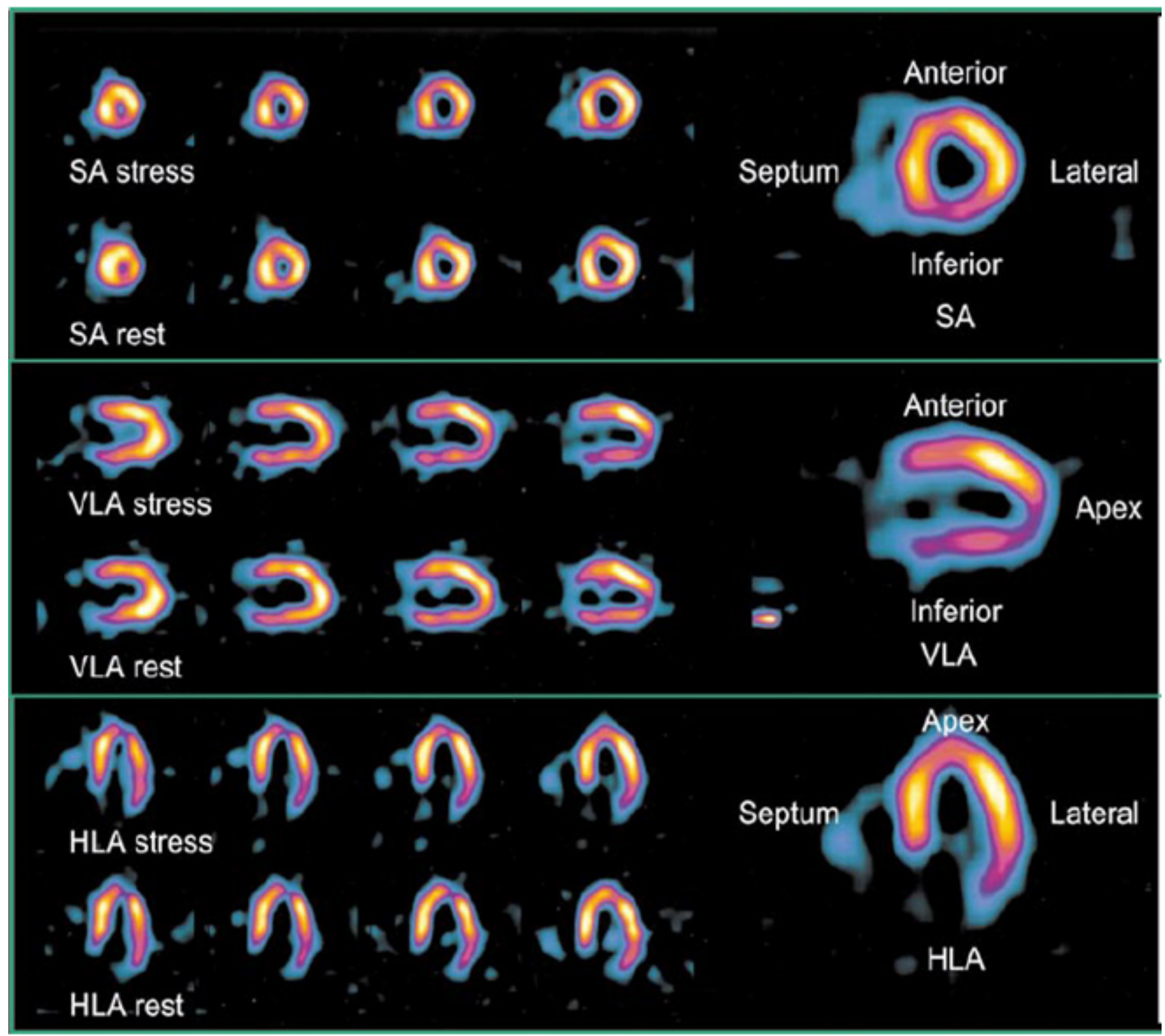

Figure 2. Myocardial perfusion visual analysis by SPECT ${ }^{16}$

unable to complete an optimal SPECT examination $(\mathrm{n}=5)$. Total of 72 patients 49 were males and mean age was $54,7 \pm 9,8$ years. The appearance of fQRS on 12-lead ECG was found in 46 patients (64\%). Most of patients with fQRS were males compared with nonfQRS group (82\% vs 42\%, p=0.001). The mean age of patients in $\mathrm{fQRS}$ group were somewhat older although the difference between two groups was not statistically significant.

From the risk factors of CAD, Type II Diabetes Mellitus (DM) and smoker were significantly different between these groups. Frequency of smoker was significantly higher in patients with fQRS (82\% vs $50 \%, \mathrm{p}=0.003) .26$ patients (56\%) with type II DM in fQRS group and 8 patients (30\%) in non-fQRS group $(\mathrm{p}=0.03)$. We found no statistically significant difference between those with and without fQRS regarding hypertension, dyslipidemia and history of PCI or CABG (Table 1).

\section{SPECT results between fQRS and non-fQRS groups}

Patients of the fQRS group had a significantly lower 

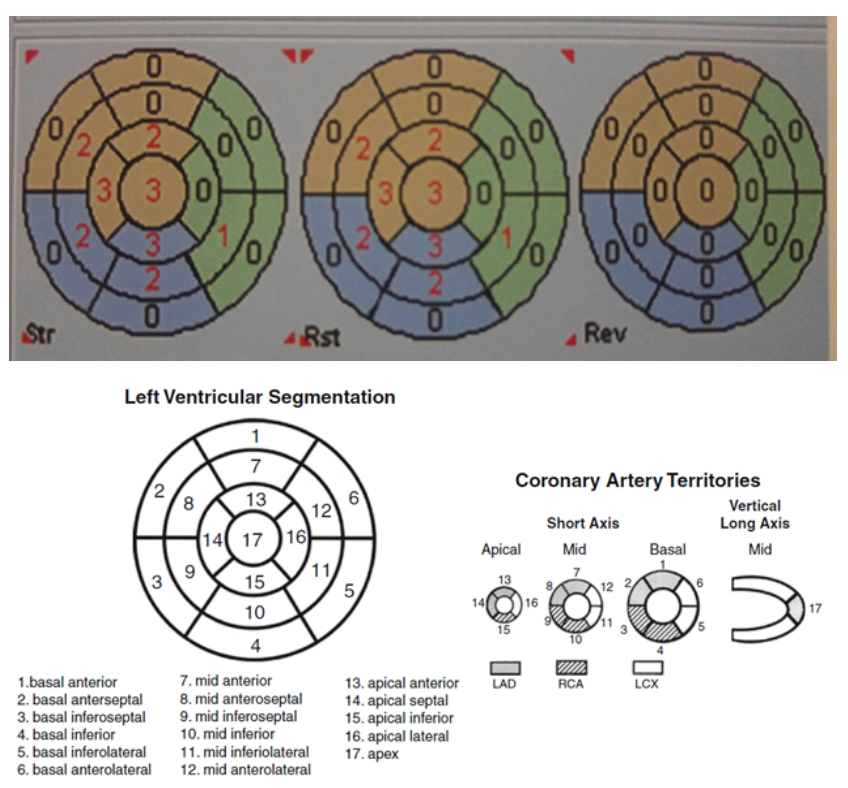

Figure 3. Semiquantitative scoring analysis on 17-segment standard nomenclature 16

Left Ventricular Ejection Fraction (LVEF) compared with non-fQRS group, $36,9 \pm 2,1 \%$ vs. $53,2 \pm 2,2$ $\%,(\mathrm{p}=0.002)$. The fQRS group had higher mean Left Ventricular End Systolic Volume (LVESV) than nonfQRS group either at resting phase or Dobutamine-load phase. The differences between the two groups were statistically significant. The LVESV at resting phase in patients with $\mathrm{fQRS}$ was $138,1 \pm 82 \mathrm{ml}$ and $97,5 \pm 64,1$ $\mathrm{ml}$ in patients without fQRS $(\mathrm{p}=0.02)$. Meanwhile, LVESV at Dobutamine-load in patients with fQRS was $139 \pm 86,1 \mathrm{ml}$ and $91 \pm 57,6 \mathrm{ml}$ in patients without fQRS ( $p=0.006)$. Incidence of myocardial scar as detected by SPECT was significantly higher in fQRS group compared with non-fQRS group. (82\% vs. $15 \%$, $\mathrm{p}<0.001$ ) (Table 2).

\section{Fragmented QRS and regional scar analysis}

Sensitivity, specificity, Positive Predictive Value (PPV) and Negative Predictive Value (NPV) of fQRS for any of myocardial scar as detected by SPECT analysis were $91 \%, 81 \%, 89 \%$, and $84 \%$, respectively. For each regional scar analysis, anterior fQRS has sensitivity, specificity, PPV and NPV of 87\%, 90\%, $91 \%$, and $85 \%$ for anterior wall. Inferior fQRS has sensitivity, specificity, PPV and NPV of 76\%, 80\%, 74\%, and $82 \%$ for inferior wall. Lateral fQRS has sensitivity, specificity,
PPV and NPV of $73 \%, 79 \%, 62 \%$, and $86 \%$ for lateral wall (Figure 4).

\section{Inter-observer variability}

The QRS Fragmentation measurement are often variably between observers. In this study, measurements of fQRS were performed by 2 observers. Inter-observer variability was measured by using Kappa test (Cohen's Kappa Coefficient). The value of Cohen's Kappa Coefficient $(\kappa)$ on inter-observer variability is 0,791 which is considered to be good agreement between the observers $(\mathrm{p}<0.001)$.

\section{Discussion}

This cross-sectional study was conducted to investigate the diagnostic capability of fragmented QRS on 12-lead ECG as a marker to detect the presence of myocardial scar and its location as examined by Stress Myocardial Perfusion Imaging (MPI) with Technetium-99m sestamibi-based SPECT. SPECT MPI is a non-invasive nuclear-based modality that has ability of localizing the location of the ischemic region and assessing the extent and severity of cardiac damage with good sensitivity and specificity ${ }^{15}$.

The patients who were either being evaluated for CAD or had a history of CAD and underwent SPECT Myocardial Perfusion Imaging were enrolled in this study. We conducted data collection through anamnesis, physical and laboratory examination, ECG, cardiac exercise test, and other supporting modalities. The risk factors for CAD (age, sex, hypertension, smoking, type II diabetes mellitus, dyslipidemia) were also investigated. We found $13 \%$ patients with fQRS had history of PCI. $8 \%$ patients with fQRS had previous CABG. No significant difference between $\mathrm{FQRS}$ and non-fQRS groups regarding the history of PCI or CABG.

In our study, from the baseline characteristics, we found 46 people (64\%) with fQRS. This did not differ greatly from the previous study that found the prevalence of $\mathrm{fQRS}$ in CAD patients was $58 \%{ }^{17}$. We observed that most of patients with $\mathrm{fQRS}$ were males. Previous studies have reported that the occurence of $\mathrm{fQRS}$ is 2-3 times higher in men than in women 4. Type II Diabetes Mellitus (DM) and smoker were the 


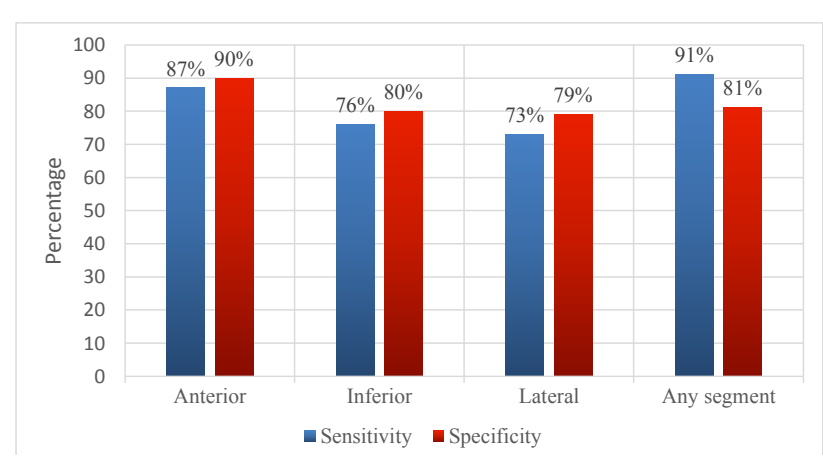

Figure 4. Sensitivity and specificity of fragmented QRS for each myocardial segment

risk factors that appeared to be significantly different between these groups.

Smoker was a significant risk factor in patients with fQRS in our study, this probably be due to patients with male-dominated fQRS, who tended to carry such risk factor. While incidence of hypertension and dyslipidemia were higher in patients with fQRS, but both were not statistically significant.

Our study findings demonstrated that fragmented QRS complexes on 12-lead ECG is a marker of myocardial perfusion abnormalities on stress SPECT study. Some previous studies have shown that fQRS became an important marker of ECG which exhibited a lower LVEF in CAD patients because its presence involves extensive areas of ischemia and myocardial damage ${ }^{18,19}$ Our study also found similar results, patients with fQRS had significantly lower LVEF when compared to patients without fQRS. In addition, the LVESV, as an important parameter of LV, was significantly greater in patients with fQRS when compared with patients without fQRS in the resting phase and Dobutamine-load phase. The LVEF, stress and rest LVESV were measured using SPECT.

Previous experimental study confirmed that after MI, significant myocar $\neg$ dial necrosis, with islands of viable myocardial tissue merge in abundant fibrous tissue. The islands of chronically ischemic myo 7 cardium display slow activation because of the partial depolarization and is probably responsi $\neg$ ble for abnormal conduction around the scarred myocardium and causing multiple spikes within QRS complexes ${ }^{12}$. The presence of fQRS is associated with an existence of myocardial scar after myocardial infarction, therefore fQRS could detect prior silent myocardial infarction and remote myocardial scar as detected by cardiac SPECT imaging 9,20 . Our study supported this finding, the incidence of myocardial scar on SPECT analysis was significantly high $\neg e r$ in patients with fQRS than in patients without fQRS. We could relate that the fragmentation of QRS complex could be a sign of myocardial scarring in CAD patients. This finding may also be the cause of lower left ventricular ejection fraction in patient with fQRS.

Some studies revealed that the initial $\mathrm{R}$-wave deflection observed is due to shifting of the QRS vector to the right and posterior as a result of early depolarization, with relatively normal conduction through the upper septum and the right ventricle in left ventricular that has a high scar load ${ }^{21,22}$. Similarly, notching or slurring in the $S$ wave may represent the activation pattern in precordial leads (leftward to the transition zone) similar to the activation pattern in left bundle-branch block. Thus, the terminal conduction delay of QRS forces arising from the area of conduction block or slowing results in an $\mathrm{fQRS}$ of ventricular activation. Therefore, different morphologies of the QRS on the surface ECG may represent various directions of myocardial activation pattern, depending on the extent and location of scar tissue in the left ventricle.

According to prior study, male sex and type II DM were the predictors of more severe stenosis, bigger infarcts size and lower left ventricular ejection fraction ${ }^{23,24}$. While other study revealed that CAD patients with fQRS appear to have a worse coronary collateralization system, thus affecting the extent of infarction ${ }^{17,25}$. Our findings are also consistent with this study, considering that $\mathrm{fQRS}$ group predominantly were males and type 2 DM patients.

From the regional scar analysis, our study found that presence of fQRS complexes on an ECG had good sensitivity, specificity, PPV and NPV for predicting the presence of a corresponding focal regional myocardial scar. The extent of global and regional perfusion abnormalities is an important predictor of fQRS complex patterns on the ECG. Moreover, patients with $\mathrm{fQRS}$ also demonstrated greater LV volumes and lower global LV systolic function. This is further reassurance to the presence of LV remodeling after MI. Hence the prompt detection of fQRS patterns on 12-lead ECG may improve our early detection of patients with suspected CAD or prior MI. 


\section{Limitations of study}

Smaller number of patients compared with previous study was the limitation of our study. Not all of the patients were diagnosed by coronary angiography as the gold standard in diagnosing CAD. In the future, further study with larger number of CAD patients who were diagnosed by coronary angiography needed, resulting in better analysis and can also determine the relationship between the fQRS on the 12-lead ECG with other parameters obtained from coronary angiography modality.

\section{Conclusions}

The fragmented QRS could serve as an important ECG marker to detect and localize the myocardial damage in patients with known or suspected CAD. Regional fQRS patterns denote the presence of regional myocardial scar and are a valuable diagnostic marker of CAD with good sensitivity and specificity.

\section{Ethical Clearance}

No: 155/TGL/KEPK FK USU-RSUP HAM/2018 from Health Research Ethical Committee, Medical Faculty of Universitas Sumatera Utara / H. Adam Malik General Hospital

\section{Publication Agreement}

The authors of this article give permission to Indonesian Journal of Cardiology (IJC) to publish this article in its journal if this article is accepted.

\section{Conflict of Interest}

The authors indicate no conflict of interest.

\section{Funding}

No external funding received by the author in order to do the research.

\section{List of Abbreviations}

CABG : Coronary Artery Bypass Grafting

CAD : Coronary Artery Disease

DM : Diabetes Mellitus

ECG : Electrocardiogram

fQRS : Fragmented QRS

LV : Left Ventricle

LVEF : Left Ventricle Ejection Fraction

LVESV : Left Ventricle End Systolic Volume

MI : Myocardial Infarction

MPI : Myocardial Perfusion Imaging

$\mathrm{ms}$ : millisecond

NPV : Negative Predictive Value

PPV : Positive Predictive Value

RISKESDAS : Riset Kesehatan Dasar

SD : Standard Deviation

SDS : Sum Difference Score

SPECT : Single Photon Emission Computed

Tomography

SRS : Sum Rest Score

SSS : Sum Stress Score

\section{References}

1. Mozaffarian D, Benjamin EJ, Go AS, et al. AHA statistical update. Heart disease and stroke statistics 2015. Update a report from the American Heart Association. Circulation. 2015; 2015:e1-e294

2. Trihono. Riset kesehatan dasar. Badan Penelitian dan Pengembangan Kesehatan Kementerian Kesehatan RI. 2013

3. Dabbagh Kakhki VR, Ayati N, Zakavi SR, et al. Comparison between frag $\neg$ mented QRS and Q waves in myocardial scar detection using myocardial perfusion single pho $\neg$ ton emission computed tomography. Kardiol Pol 2015; 73:437-44.

4. Ozdemir S, Tan YZ, Colkesen Y, et al. Comparison of fragmented QRS and myocardial perfusion-gated SPECT findings. Nucl Med Commun. 2013; 34:1107-15.

5. Yasuda M, Iida H, Itagane $\mathrm{H}$ et al. Significance of Q wave disappearance in the chronic phase following transmural acute myocardial infarction. Jpn Circ J, 1990; 54:1517-24.

6. Das MK, Michael MA, Suradi H, et al. Usefulness of fragmented QRS on a 12-lead electrocardiogram 
in acute coronary syndrome for predicting mortality. Am J Cardiol 2009; 104:1631-37.

7. Pietrasik G, Zareba W. QRS fragmentation: diagnostic and prognostic significance. Cardiol J 2012; 19:114-21

8. Flowers NC, Horan LG, Thomas JR, et al. The anatomic basis for high-frequency components in the electrocardiogram. Circulation. 1969;39:531-9.

9. Das MK, B. Khan, S. Jacob, et al. "Significance of a Fragmented QRS Complex Versus a Q Wave in Patients with Coronary Artery Disease." Circulation. 2006;113(21);2495-501.

10. Chatterjee S, Changawala N. Fragmented QRS Complex: a novel marker of cardiovascular dis $\neg$ ease. Clin Cardiol. 2010;33:68-71.

11. Das MK, Saha C, El Masry H, et al. Fragmented QRS on a 12-lead ECG: a predictor of mortality and cardiac events in patients with coronary artery disease. Heart Rhythm. 2007; 4:1385-92.

12. Gardner PI, Ursell PC, Fenoglio JJ Jr, et al. Electrophysiologic and anatomic basis for fractionated electrograms recorded from healed myocardial infarcts. Circulation. 1985; 72:596-611.

13. Driver KA, Atchley AE, Kaul P, et al. Single photon emission computed tomography myocardial imaging: clinical applications and future directions. Minerva Cardioangiol. 2009;57:333-47.

14. Sharir T, Berman DS, Waechter PB, et al. Quantitative analysis of regional motion and thickening by gated myocardial perfusion SPECT: normal heterogeneity and criteria for abnormality. J Nucl Med. 2001;42:1630-8.

15. Hachamovitch R, Berman DS. New frontiers in risk stratification using stress myocardial perfusion single photon emission computed tomography. Curr Opin Cardiol 2003;18:494-502.

16. Cerqueira MD, Weissman NJ, Dilsizian V, et al. Standardized myocardial segmentation and nomenclature for tomographic imaging of the heart: a statement for healthcare professionals from the Cardiac Imaging Committee of the Council on Clinical Cardiology of the American Heart Association. Circulation. 2002;105:539-42.

17. Bonakdar H, Moladoust H, Kheirkhah J, et al. Significance of a fragmented QRS complex in patients with chronic total occlusion of coronary ar- tery without prior myocardial infarction. Anatol J Cardiol. 2015;15(0):000-000

18. Canga A, Kocaman SA, Durakoğlugil M, et al. Relationship between fragmented QRS complexes and left ventricular systolic and diastolic functions. Herz 2013;38:665-70.

19. Ma $\mathrm{Y}$, de Castro Brás LE, Toba $\mathrm{H}$, et al. Myofibroblasts and the extracellular matrix network in post-myocardial infarction cardiac remodeling. Pflugers Arch. 2014; 466:1113-27

20. Mahenthiran J, Khan BR, Sawada SG, et al. Fragmented QRS complexes not typical of a bundle branch block: a marker of greater myocardial perfusion tomography abnormalities in coronary artery disease. J Nucl Cardiol. 2007;14(3):347-53

21. El-Sherif $N$. The rsR' pattern in left surface leads in ventricular aneurysm. Br Heart J. 1970;32:440-8.

22. Wiener I, Mindich B, Pitchon R. Fragmented endocardial electrical activity in patients with ventricular tachycardia: a new guide to surgical therapy. Am Heart J. 1984;107:86 -90.

23. Arad Y, Newstein D, Cadet F, et al. Association of multiple risk factors and insulin resistance with increased prevalence of asymptomatic coronary artery disease by an electron-beam computed tomographic study. Arteriosclerosis Thrombosis and Vascular Biology. 2001;21:2051-8

24. Natali A, Vichi S, Landi P, et al. Coronary atherosclerosis in Type II diabetes: angiographic findings and clinical outcome. Diabetologia. 2000;43:63241

25. Erdogan T, Kocaman SA, Cetin M, et al. Relationship of fragmented QRS complexes with inadequate coronary collaterals in patients with chronic total occlusion. J Cardiovasc Med (Hagerstown) 2012; 13:499-504 